\section{Lessons Learned from the Tokyo Subway Sarin Attack}

Tetsu Okumura; Kouichiro Suzuki; Shinichi Ishimatsu; Nobukatsu Takasu; Chiibo Fuiji; Akitsugu Kobama

Department of Acute Medicine, Kawasaki Medical School Hospital, Kurashiki-City, Okayama, JAPAN

On the morning of 20 March 1995, sarin was released in the Tokyo Subway System. There had never been such a large scale act of urban terrorism using a nerve gas. There are many lessons to be learned from Tokyo Subway Sarin Attack. Two major lessons can be cited in summary:

1) Absence of decontamination - In total, 1,364 EMTs were dispatched, and among them, 135 were secondarily affected. At St. Luke's hospital, $23 \%$ of the medical staff complained of symptoms and signs of secondary exposure. Fortunately, nobody died from the secondary exposure. The religious cult used a $30 \%$ sarin solution. If they had used a $100 \%$ sarin solution, the outcome would have been much more tragic - secondarily exposed prehospital and medical staff would have been killed. This is the reason for the development of decontamination facilities and the use of personal protective equipment (PPE) in the prehospital and hospital settings; and

2) Confusion of information and lack of coordination among related organizations - Japan is a highly vertically structured society. Fire departments, police, metropolitan governments, and hospitals acted independently without coordination. After the attack, the Japanese government developed the Severe Chemical Hazard Response Team.

The Prime Minister's office created a National Security and Crisis Management Office that calls realistic desktop hazmat drills involving the concerned organizations and specialists.

Keywords: chemicals; communications; contamination; coordination; decontamination; hazardous materials (hazmat); hospitals; prehospital; sarin; subway; terrorism; Tokyo

E-mail: xj2t-okmr@asahi-net.or.jp

\section{Disaster Coordination and Management Experiences in Costa Rica \\ Daniel L. Rodriguez, MD}

Department of Medicine, Hospital Calderon Guardia, Universidad de Costa Rica, San José, COSTA RICA

To reduce morbidity and mortality due to disasters, it is necessary to intervene in a timely and integral manner in each of disasters phases. Knowledge and awareness of risks and vulnerability should lead to interventions that would prevent disasters or mitigate and prepare for their consequences.

A multi-disciplinary team, responsible for planning, organising, and implementing disaster management not only should have expertise and experience in the field, but also legal authority, as well as financial and political support. Multidisciplinary teams at institutional, local, regional, sectorial, and national levels, with excellent communications and a clear chain of command, must coordinate operations according to previously designed and approved protocols. Integrated and feasible plans by simulations, drills, and management of daily emergencies, if a timely, organized, must be generated from response and an efficient utilization of financial, material, and human resources is desired.

In current legislation, in Costa Rica the creation of the National Emergency Commission, the approval and implementation of national, sectorial, and institutional plans and the establishment of local, regional, and national committee funds to face disasters, as well as public awareness, and education have contributed to a better management of disaster situations, and interventions leading to preventing and mitigating their consequences. Keywords: awareness; committees; Costa Rica; disasters; emergency management; legislation; resources; support; team 